\title{
Generalized Ricci solitons on $K$-contact manifolds
}

\author{
Gopal Ghosh* and Uday Chand De
}

\begin{abstract} an Einstein one. Finally, we obtain several remarks.

Keywords: K-contact manifold; Generalized Ricci soliton; Einstein manifold.

AMS Subject Classification (2020): Primary: 53C15; 53C55.

${ }^{*}$ Corresponding author
\end{abstract}

The object of the present paper is to study $K$-contact manifold admitting generalized Ricci solitons. We prove that a $K$-contact manifold of dimension $(2 n+1)$ satisfying the generalized Ricci soliton equation is

\section{Introduction}

Let $M$ be a $(2 n+1)$-dimensional differentiable manifold. Suppose that $(\phi, \xi, \eta, g)$ is an almost contact metric structure on $M$. This means that $(\phi, \xi, \eta, g)$ is a quadruple consisting of a $(1,1)$-tensor field $\phi$, an associated vector field $\xi$, a 1-form $\eta$ and a Riemannian metric $g$ on $M$ satisfying the following relations

$$
\phi^{2}(X)=-X+\eta(X) \xi, \quad \eta(\xi)=1, \quad g(\phi X, \phi Y)=g(X, Y)-\eta(X) \eta(Y),
$$

where $X, Y$ are smooth vector fields on $M$. In addition, we have

$$
\phi \xi=0, \eta(\phi X)=0, g(X, \xi)=\eta(X), g(\phi X, Y)=-g(X, \phi Y) .
$$

An almost contact structure is said to be a contact structure if $g(X, \phi Y)=d \eta(X, Y)$. A contact metric structure is said to be normal if the induced almost complex structure $J$ on the product manifold $M^{2 n+1} \times \mathbb{R}$ defined by

$$
J\left(X, f \frac{d}{d t}\right)=\left(\phi X-f \xi, \eta(X) \frac{d}{d t}\right)
$$

is integrable where $X$ is tangent to $M, t$ is the coordinate of $\mathbb{R}$ and $f$ is a smooth function on $M^{2 n+1} \times \mathbb{R}$. A normal contact metric manifold is called a Sasakian manifold. If $\xi$ is a Killing vector field on a contact metric manifold $(M, g)$, then the manifold is called a $K$-contact metric manifold or simply a $K$-contact manifold ([1], [15]). An almost contact manifold is Sasakian [1], if and only if

$$
\left(\nabla_{X} \phi\right)(Y)=g(X, Y) \xi-\eta(Y) X
$$

where $\nabla$ is the Levi-Civita connection.

A complete regular contact metric manifold $M^{2 n+1}$ carries a $K$-contact structure $(\phi, \xi, \eta, g)$, defined in terms of the almost Kähler structure $(J, G)$ of the base manifold $M^{2 n+1}$. Here the $K$-contact structure $(\phi, \xi, \eta, g)$ is Sasakian if and only if the base manifold $\left(M^{2 n+1}, J, G\right)$ is Kählerian. If $\left(M^{2 n+1}, J, G\right)$ is only almost Käehler, then $(\phi, \xi, \eta, g)$ is only $K$-contact [1]. In a Sasakian manifold, the Ricci operator $Q$ commutes with $\phi$, that is, $Q \phi=\phi Q$. Recently in [11], it has been shown that there exist $K$-contact manifolds with $Q \phi=\phi Q$ which are not Sasakian. It is seen that $K$-contact structure is the intermediate between contact and Sasakian structure. $K$-contact manifolds have

Received : 02-02-2020, Accepted : 17-10-2010 
been studied by several authors ([6], [7], [8], [14], [16], [18]) and many others. Given a smooth function $f$ on $M$, the gradient of $f$ is defined by

$$
g(\operatorname{grad} f, X)=X f
$$

the Hessian of $f$ is defined by

$$
(\text { Hess } f)(X, Y)=g\left(\nabla_{X} \operatorname{grad} f, Y\right),
$$

for all smooth vector fields $X, Y$. For a smooth vector field $X$, we have ([12], [13])

$$
X^{b}(Y)=g(X, Y) .
$$

The generalized Ricci soliton equation in a Riemannian manifold $(M, g)$ is defined by [13]

$$
£_{X} g=-2 c_{1} X^{b} \odot X^{b}+2 c_{2} S+2 \lambda g,
$$

where $£_{X} g$ is the Lie derivative of $g$ along $X$ given by

$$
\left(£_{X} g\right)(Y, Z)=g\left(\nabla_{Y} X, Z\right)+g\left(\nabla_{Z} X, Y\right),
$$

for all smooth vector fields $X, Y, Z$ and $c_{1}, c_{2}, \lambda \in \mathbb{R}$. For different values of $c_{1}, c_{2}$ and $\lambda$, equation (1.7) is a generalization of Killing equation $\left(c_{1}=c_{2}=\lambda=0\right)$, equation for homotheties $\left(c_{1}=c_{2}=0\right)$, Ricci soliton $\left(c_{1}=0, c_{2}=-1\right)$, Vacuum near-horizon geometry equation $\left(c_{1}=1, c_{2}=\frac{1}{2}\right)$ etc. For more details we refer to the reader ([3], [4], [5], [9], [13]).

If $X=\operatorname{grad} f$, then the generalized Ricci soliton equation is given by

$$
\text { Hess } f=-c_{1} d f \odot d f+c_{2} S+\lambda g .
$$

\section{Preliminaries}

In an $(2 n+1)$-dimensional $K$-contact manifold, the following relations hold ([1], [17])

$$
\begin{gathered}
\nabla_{X} \xi=-\phi X \\
g(R(\xi, X) Y, \xi)=\eta(R(\xi, X) Y)=g(X, Y)-\eta(X) \eta(Y), \\
R(\xi, X) \xi=-X+\eta(X) \xi \\
S(X, \xi)=2 n \eta(X), \\
\left(\nabla_{X} \phi\right) Y=R(\xi, X) Y,
\end{gathered}
$$

for any vector fields $X, Y \in \chi(M)$.

A $K$-contact manifold $M$ of dimension $\geq 3$ is said to be Einstein if its Ricci tensor $S$ is of the form $S=a g$, where $a$ is a constant.

In this case we have

$$
S(X, Y)=a g(X, Y) .
$$

Substituting $X=Y=\xi$ in (2.6) and then using (2.4) and (1.2), we get

$$
a=2 n .
$$

Thus using (2.7) we obtain from (2.6)

$$
S(X, Y)=2 n g(X, Y)
$$

Again from (2.8) we infer that

$$
Q X=2 n X .
$$




\section{Generalized Ricci soliton}

In this section we characterize $K$-contact manifolds admitting generalized Ricci soliton. First we prove the following Lemma:

Lemma 3.1. Let $(M, \phi, \xi, \eta, g)$ be a K-contact manifold. Then

$$
\left(£_{\xi}\left(£_{X} g\right)\right)(Y, \xi)=g(X, Y)+g\left(\nabla_{\xi} \nabla_{\xi} X, Y\right)+Y g\left(\nabla_{\xi} X, \xi\right),
$$

for all smooth vector fields $X, Y$ with $Y$ orthogonal to $\xi$.

Proof. We have

$$
\begin{aligned}
& \left(£_{\xi}\left(£_{X} g\right)\right)(Y, \xi)=\xi\left(\left(£_{X} g\right)(Y, \xi)\right)-\left(£_{X} g\right)\left(£_{\xi} Y, \xi\right)-\left(£_{X} g\right)\left(Y, £_{\xi} \xi\right) \\
& =\xi\left(\left(£_{X} g\right)(Y, \xi)\right)-\left(£_{X} g\right)\left(£_{\xi} Y, \xi\right) .
\end{aligned}
$$

Using (1.8) in (3.1) yields

$$
\begin{aligned}
& \left(£_{\xi}\left(£_{X} g\right)\right)(Y, \xi)=\xi g\left(\nabla_{Y} X, \xi\right)+\xi g\left(\nabla_{\xi} X, Y\right)-g\left(\nabla_{[\xi, Y]} X, \xi\right) \\
& -g\left(\nabla_{\xi} X,[\xi, Y]\right)=g\left(\nabla_{\xi} \nabla_{Y} X, \xi\right)+g\left(\nabla_{Y} X, \nabla_{\xi} \xi\right)+g\left(\nabla_{\xi} \nabla_{\xi} X, Y\right) \\
& +g\left(\nabla_{\xi} X, \nabla_{\xi} Y\right)-g\left(\nabla_{[\xi, Y]} X, \xi\right)-g\left(\nabla_{\xi} X, \nabla_{\xi} Y\right)+g\left(\nabla_{\xi} X, \nabla_{Y} \xi\right) \\
& =g\left(\nabla_{\xi} \nabla_{Y} X, \xi\right)+g\left(\nabla_{Y} X, \nabla_{\xi} \xi\right)+g\left(\nabla_{\xi} \nabla_{\xi} X, Y\right)-g\left(\nabla_{[\xi, Y]} X, \xi\right) \\
& +g\left(\nabla_{\xi} X, \nabla_{Y} \xi\right) .
\end{aligned}
$$

Now by the definition of Riemannian curvature tensor, from (3.2) it follows that

$$
\left(£_{\xi}\left(£_{X} g\right)\right)(Y, \xi)=g(R(\xi, Y) X, \xi)+g\left(\nabla_{\xi} \nabla_{\xi} X, Y\right)+Y g\left(\nabla_{\xi} X, \xi\right) .
$$

Using (2.2) in (3.3) and with $Y$ orthogonal to $\xi$, we infer that

$$
\left(£_{\xi}\left(£_{X} g\right)\right)(Y, \xi)=g(X, Y)+g\left(\nabla_{\xi} \nabla_{\xi} X, Y\right)+Y g\left(\nabla_{\xi} X, \xi\right) .
$$

Lemma 3.2. [12] Let $(M, g)$ be a Riemannian manifold and let $f$ be a smooth function. Then

$$
\left(£_{\xi}(d f \odot d f)\right)(Y, \xi)=Y(\xi(f)) \xi(f)+Y(f) \xi(\xi(f)),
$$

for every vector field $Y$.

Lemma 3.3. Let $(M, \phi, \xi, \eta, g)$ be a K-contact manifold which satisfies the generalized Ricci soliton equation. Then

$$
\nabla_{\xi} \operatorname{grad} f=\left(\lambda+2 c_{2} n\right) \xi-c_{1} \xi(f) \operatorname{grad} f .
$$

Proof. Using (2.4) we have

$$
\lambda \eta(Y)+c_{2} S(\xi, Y)=\left(\lambda+2 c_{2} n\right) \eta(Y) .
$$

Making use of (1.9) and (3.4) implies

$$
(\text { Hess } f)(\xi, Y)=-c_{1} \xi(f) g(\operatorname{grad} f, Y)+\left(\lambda+2 c_{2} n\right) \eta(Y) .
$$

Hence the Lemma follows from (3.5) and the definition of the Hessian (1.9).

Now we prove our main theorem as follows:

Theorem 3.1. Suppose that $(M, \phi, \xi, \eta, g)$ is a $K$-contact manifold of dimension $(2 n+1)$ which satisfies the generalized gradient Ricci soliton equation with $c_{1}\left(\lambda+2 c_{2} n\right) \neq-1$. Then $f$ is a constant function. Furthermore, if $c_{2} \neq 0$, then the manifold is an Einstein one. 
Proof. Suppose that $Y$ is orthogonal to $\xi$. Then from Lemma 3.1 with $X=\operatorname{grad} f$, we have

$$
2\left(£_{\xi}(\text { Hess } f)\right)(Y, \xi)=Y(f)+g\left(\nabla_{\xi} \nabla_{\xi} \operatorname{grad} f, Y\right)+Y g\left(\nabla_{\xi} \operatorname{grad} f, \xi\right) .
$$

Using Lemma 3.3 in (3.6) yields

$$
\begin{aligned}
& 2\left(£_{\xi}(\text { Hess } f)\right)(Y, \xi)=Y(f)+\left(\lambda+2 c_{2} n\right) g\left(\nabla_{\xi} \xi, Y\right) \\
& -c_{1} g\left(\nabla_{\xi}(\xi(f) \operatorname{grad} f), Y\right)+\left(\lambda+2 c_{2} n\right) Y-c_{1} Y\left(\xi(f)^{2}\right) \\
& =Y(f)-c_{1} g\left(\nabla_{\xi}(\xi(f) \operatorname{grad} f), Y\right)+\left(\lambda+2 c_{2} n\right) Y-c_{1} Y\left(\xi(f)^{2}\right) .
\end{aligned}
$$

Again using Lemma 3.3 with $Y$ orthogonal to $\xi$, from (3.7) it follows that

$$
\begin{aligned}
& 2\left(£_{\xi}(\text { Hess } f)\right)(Y, \xi)=Y(f)-c_{1} \xi(\xi(f)) Y(f)+c_{1}^{2} \xi(f)^{2} Y(f) \\
& -2 c_{1} \xi(f) Y(\xi(f)) .
\end{aligned}
$$

Since $\xi$ is a Killing vector field, so $£_{\xi} g=0$, it implies $£_{\xi} S=0$. Using the above fact and taking the Lie derivative to the generalized Ricci soliton equation (1.9) yields

$$
2\left(£_{\xi}(\text { Hess } f)\right)(Y, \xi)=-2 c_{1}\left(£_{\xi}(d f \odot d f)\right)(Y, \xi) .
$$

Using (3.8), (3.9) and Lemma 3.2 we infer that

$$
Y(f)\left[1+c_{1} \xi(\xi(f))+c_{1} \xi\left(f^{2}\right)\right]=0 .
$$

According to Lemma 3.3 we have

$$
\begin{aligned}
& c_{1} \xi(\xi(f))=c_{1} \xi g(\xi, \operatorname{grad} f) \\
& =c_{1} g\left(\xi, \nabla_{\xi} \operatorname{grad} f\right) \\
& =c_{1}\left(\lambda+2 c_{2} n\right)-c_{1}^{2} \xi(f)^{2} .
\end{aligned}
$$

Making use of (3.10) and (3.11), we obtain

$$
Y(f)\left[1+c_{1}\left(\lambda+2 c_{2} n\right)\right]=0,
$$

which implies

$$
Y f=0,
$$

provided $1+c_{1}\left(\lambda+2 c_{2} n\right) \neq 0$. Therefore, $\operatorname{grad} f$ is parallel to $\xi$. Hence $\operatorname{grad} f=0$ as $d=\operatorname{ker} \eta$ is nowhere integrable, that is, $f$ is a constant function. Thus the manifold is an Einstein one follows from (1.9).

Remark 3.1. We know that [10] every Sasakian manifold is $K$-contact, but the converse is not true in general. However, a 3-dimensional $K$-contact manifold is Sasakian. Thus our main Theorem 3.1 is the generalization of Theorem 1.1 of [12].

Remark 3.2. Since a compact $K$-contact Einstein manifold is Sasakian [2], therefore a compact $K$-contact manifold admitting generalized Ricci solitons is Sasakian.

\section{Acknowledgements}

The authors are grateful to the referees for their valuable comments and suggestions.

\section{References}

[1] Blair, D.E.: Contact manifolds in Reimannian geometry. Lecture notes in Math. 509, Springer-Verlag. (1976).

[2] Boyer, C.P., Galicki, K.: Einstein manifold and contact geometry, Proc. Amer. Math. Soc. 129, 2419-2430 (2001). 
[3] Chrusciel, P.T., Reall, H.S., Tod, P.: On non-existence of static vacuum black holes with degenerate components of the event horizon. Classical Quantum Gravity, 23, 549-554 (2006).

[4] Deshmukh, S., Aloden, H.: A note on Ricci soliton. Balkan J. Geom. Appl. 16 , 48-55 (2011).

[5] Deshmukh, S.: Jacobi-type vector fields on Ricci solitons. Bull. Mathematique de la Societe des Sciences Mathematiques de Roumanie Nouvelle Series. 103, 41-50 (2012).

[6] De, U.C., Biswas, S.: On K-contact $\eta$-Einstein manifolds. Bull. Soc. Math. 16, 23-28 (1990).

[7] De, U.C., De, A.: On some curvature properties of K-contact manifolds. Extracta Math. 27, 125-134 (2012).

[8] Guha, N., De, U.C.: On K-contact manifolds. Serdica-Bulgaricae Math. Publ. 19, 267-272 (1993).

[9] Jezierski, J.: On the existance of Kundts metrics and degenerate (or extremal) Killing horizones. Classical Quantum Gravity, 26, 035011, 11pp (2009).

[10] Jun, J.B., Kim, U. K.: On 3-dimensional almost contact metric manifolds. Kyungpook Math. J. 34, 293-301 (1994).

[11] Koufogiorgos, T.: Contact metric manifolds. Ann. Global Anal. Geom. 11, 25-34 (1993).

[12] Mekki, M.E., Cherif, A.M.: Generalised Ricci solitons on Sasakian manifolds. Kyungpook Math. J. 57 677-682 (2017).

[13] Nurowski. P., Randall, M.: Generalised Ricci solitons. J. Geom. Anal. 26, 1280-1345 (2016).

[14] Prasad, R., Srivastava, V.: On $\phi$-symmetric K-contact manifolds. IJRRAS. 16, 104-110 (2013).

[15] Sasaki, S.: Lecture notes on almost contact manifolds. Part I. Tôhoku Univ. (1965).

[16] Tarafdar, D., De, U.C.: On K-contact manifolds. Bull. Math. Soc. Sci. Math. Roumanie. 37, 207-215 (1993).

[17] Yano, K., Kon, M.: Structures on manifolds. World Scientific Press. Vol 40, (1989).

[18] Yildiz, A., Ata, E.: On a type of K-contact manifolds. Hacettepe J. Math. Stat. 41, 567-571 (2012).

\section{Affiliations}

GOPAL GHOSH

AdDRESS: University of Calcutta, Dept. of Pure Mathematics, 700019, West Bengal-India.

E-MAIL: ghoshgopal.pmath@gmail.com

ORCID ID: 0000-0001-6178-6340

UDAY CHAND DE

AdDress: University of Calcutta, Dept. of Pure Mathematics, 700019, West Bengal-India.

E-MAIL: Uc_de@yahoo.com

ORCID ID: 0000-0002-8990-4609 